\title{
Closing the health gap among Canadians: Using Co-Active Life Coaching to address the challenges to primary healthcare
}

\author{
Rebecca Liu
}

\section{University of Western Ontario}

Several studies have reported the burden of obesity and cardiometabolic risk to be disproportionately greater among ethnic minorities in comparison to those of European-descent. ${ }^{1,2}$ Generally, the "healthy immigrant effect' is proposed as the reason for this disparity, in which new immigrants start to lose their health advantage when they start to adopt the physical, social and cultural environment of their newly adopted country. ${ }^{1}$ However, evidence suggests that aboriginal populations also experience poorer obesity-related health outcomes relative to the entire Canadian population, ${ }^{3}$ which suggests that this health inconsistency may not be exclusive to immigrant populations. Additionally, this may reflect a deeper issue of accessibility and utilization of primary care services among Canada's diverse population. The following paper will explore the barriers to healthcare access and utilization, specifically primary care among ethnic minorities. In addition, this paper aims to highlight a potential health behaviour intervention, known as Co-Active Life Coaching (CALC), which may serve as a means to manage obesity and cardiometabolic risk and ultimately, alleviate the high demand for primary care.

\section{Health needs unmet: A brief explanation of the challenges}

Disparities in primary healthcare accessibility and utilization have been reported among differentethnic minority groups. ${ }^{4}$ A limited number of primary care physicians dispersed among aboriginal communities tend to carry large patient loads. ${ }^{4}$ Thus, healthcare providers working in these remote areas are unable to devote sufficient time, resources, and ultimately, the accessibility needed for their patients. Furthermore, researchers also noted the main reasons for under-utilization among a sample of ethnic minorities to be a combination of timely treatment, language barriers, and a preference for alternative or traditional medicines. ${ }^{5}$ For instance, $59 \%$ of an East Asian minority group indicated a preference for native language speaking physicians, with only $38 \%$ of those participants actually obtaining a native-speaking provider. ${ }^{5}$ Despite some regions possessing adequate accessibility to healthcare services, barriers continue to persist among ethnic minorities. Therefore, the need for a remotely accessible, more timely, and language/ culturally supportive assistance is essential to increase healthcare utilization and to meet the health demands of Canada's population.

\section{A need for mobile health: What can be done?}

The existence of a comprehensive health intervention may be able to mitigate the health status gap among Canadians struggling with obesity and cardiometabolic risk, and to address some of the primary care challenges. Health coaching, which focuses on facilitating personal healthrelated topics and goals, has become more commonly utilized in treating cardiometabolic and obesity-related conditions such as hypertension, depression, diabetes, cardiovascular health, and asthma. ${ }^{6} \mathrm{CALC}$ is a form of health coaching that is commonly telecommunicated and which has been utilized to effectively alleviate several obesityrelated conditions. ${ }^{7,8}$ Theoretically grounded ${ }^{9}$ and evidencebased, ${ }^{7,8}$ this behavioural intervention can be conducted by a variety of health professionals such as dieticians, nurses, and/or social workers. It is used to explore the client's health needs and empower clients to develop solutions that best suit his/her lifestyle long-term. ${ }^{9}$ CALC can be geared towards ameliorating the challenges of remote accessibility, more timely care, and language/culturally supportive assistance among Canada's diverse population. Firstly, CALC via telecommunication provides a complementary healthcare service option in remote areas. This can either be used as an initial screen and/or as a follow-up to primary care, if health services are limited in the area. Secondly, it can reduce physicians' patient load and wait times such that patients will have undergone CALC sessions prior to or as a followup to their visit, consequently allowing physicians to be 
more efficient in their health assessments. It will also help to utilize other community-based family health practitioners in the area since this type of intervention is not limited to being administered by physicians. Lastly, CALC provides the opportunity for translators or ethnic minority healthcare providers, who may not be geographically accessible, to be better positioned to understand the cultural health needs of their patients and offer traditional medical alternatives. Evidence of interpreter services used in the delivery of healthcare has demonstrated increases in patient use of primary health services. ${ }^{10}$ Therefore, incorporating several interpreters may be a viable option to overcome language and cultural barriers in different communities. However, a few limitations of this health intervention warrant mention. CALC is not a substitute for medical care, such that if a serious health condition arises, a physician should be contacted immediately. Thus, this only moderately addresses the challenges of accessibility for urgent primary care. As well, since it is an emerging technique, it is difficult to assess whether there are sufficient certified CALC coaches available for language/cultural-sensitive assistance. Ultimately, CALC is a complementary health aid to primary healthcare, which may improve the much-needed mobility for ethnic minority communities. Incorporating and piloting this health intervention into diverse communities may steadily bridge the health gap among ethnic minority Canadians.

\section{References}

1. Setia MS, Quesnel-Vallee A, Abrahamowicz M, Tousignant $P$, Lynch J. Access to health-care in Canadian immigrants: a longitudinal study of the National Population Health Survey. Health Soc Care Community. 2011;19(1):70-9.

2. Liu R, So L, Mohan S, Khan N, King K, Quan H. Cardiovascular risk factors in ethnic populations within Canada: results from national cross-sectional surveys. Open Med. 2010;4(3):e143-53.

3. Razak F, Anand S, Vuksan V. Ethnic differences in the relationships between obesity and glucose-metabolic abnormalities: a cross-sectional populationbased study. Int J Obes. 2005;29(6):656-67.

4. Shah B, Gunraj N, Hux J. Markers of access to and quality of primary care for aboriginal people in Ontario, Canada. Am J Public Health. 2003;93(5):798-802.

5. Wang L, Rosenberg M, Lo L. Ethnicity and utilization of family physicians: A case study of Mainland Chinese immigrants in Toronto, Canada. Soc Sci Med. 2008;67(9):1410-22.

6. Ammentorp J, Uhrenfeldt L. Can life coaching improve health outcomes?-A systematic review of intervention studies. BMC Health Serv Res. 2013;13:428-38.

7. Newnham-Kanas C, Irwin JD, Morrow D. Co-active life coaching as a treatment for adults with obesity. Int J Evid Based Coach Mentor. 2008;6:1-12.

8. Zandvoort M Van, Sciences $H$, Ontario W. The impact of Co-active Life Coaching on female university students with obesity. Int J Evid Based Coach Mentor. 2009;7:104-19.

9. Irwin J, Morrow D. Health promotion theory in practice: an analysis of CoActive Coaching. Int J Evid Based Coach Mentor. 2005;3(1):29-38.

10. Jacobs $E$, Lauderdale D. Impact of interpreter services on delivery of health care to limited-English-proficient patients. J Gen Intern Med. 2001;16(7):468-74.

\section{Rebecca Liu}

Rebecca H. Liu completed her B.Sc. (Honours) in Life Sciences from Queen's University and her MSc in Kinesiology from York University. She is currently a first-year PhD student in Health \& Rehabilitation Sciences (Health Promotion) at the University of Western Ontario. Her research focuses on using Co-Active Life Coaching (CALC) as a health behavioural intervention to reduce cardiometabolic risk among racial/ethnic minority groups struggling with obesity. She is also interested on investigating the impact of CALC as a psychosocial support before and after weight reduction surgery among bariatric surgical patients. 